The norm of the number on the left is found to be $p$. It seems impracticable to determine whether or not $p$ has actual prime factors in the field of 128 th roots of 1 , but this is very improbable, as the class number in that field is a multiple of 21,121 .*

The use of complex numbers appears to be of no assistance in the problem of determining whether $F_{n}$ is prime or composite.

\title{
AN EXTENSION OF CERTAIN INTEGRABILITY CONDITIONS.
}

BY PROFESSOR J. EDMUND WRIGHT.

Suppose there are $n$ functions $a_{1}, a_{2}, \ldots, a_{n}$ of $n$ independent variables $x_{1}, x_{2}, \cdots, x_{n}$, satisfying the conditions

$$
\frac{\partial a_{p}}{\partial x_{q}}-\frac{\partial a_{q}}{\partial x_{p}}=0
$$

for all values of $p$ and $q$. It is well known that the functions $a$ must all be first derivatives of a single function $V$. Similarly, if there are $\frac{1}{2} n(n+1)$ functions $a_{p^{n}}$ such that $a_{p^{n}}=a_{q p}$, satisfying the relations

$$
\frac{\partial a_{p q}}{\partial x_{r}}=\frac{\partial a_{p r}}{\partial x_{q}}
$$

for all values of $p, q, r$, then the $a$ 's must be second derivatives of a single function.

The following question arises in connection with an application of the theory of invariants of quadratic differential forms :

Suppose there are $n(n+1)$ functions $H_{p q}, K_{p q}$ such that $H_{p q}=H_{q p}, K_{p q}=K_{q p}$, satisfying the conditions

$$
\frac{\partial}{\partial x_{r}}\left(H_{p q}\right)+K_{p q} \frac{\partial Y}{\partial x_{r}}=\frac{\partial}{\partial x_{p}}\left(H_{q r}\right)+K_{q r} \frac{\partial Y}{\partial x_{p}},
$$

for all values of $p, q, r ; Y$ being a given function of the variables ; what are the conditions on the functions $H, K$ ?

We first consider the case of $2 n$ functions $a_{1}, a_{2}, \ldots, a_{n} ; b_{1}$, $b_{2}, \cdots, b_{n}$, satisfying the conditions

\footnotetext{
* Reuschle, Tafeln, p. 461.
} 


$$
\frac{\partial a_{p}}{\partial x_{q}}-\frac{\partial a_{q}}{\partial x_{p}}=b_{q} \frac{\partial Y}{\partial x_{p}}-b_{p} \frac{\partial Y}{\partial x_{q}} .
$$

Take three equations of the type (1), those for $(p, q),(q, r)$, $(r, p)$, differentiate the first with respect to $r$, the second with respect to $p$, the third with respect to $q$, and add. The quantities $a$ are eliminated, and we have the result

$$
\left(b_{q r}-b_{r q}\right) Y_{p}+\left(b_{r p}-b_{p r}\right) Y_{q}+\left(b_{p q}-b_{q p}\right) Y_{r}=0
$$

where additional suffixes denote differentiation.

Now the equations (1) are unaltered if we replace $b_{p}$ by $b_{p}^{\prime}+\lambda Y$, where $\lambda$ is an arbitrary function of the variables, and functions $b$ and $\lambda$ can be determined to satisfy the two equations

$$
b_{1}=\lambda Y_{1}+\frac{\partial b}{\partial x_{1}}, \quad b_{2}=\lambda Y_{2}+\frac{\partial b}{\partial x_{2}},
$$

for elimination of $\lambda$ gives a single equation for $b$, and any solution of this, combined with one of the above equations serves to determine $\lambda$.

We may thus in equations (1), (2), assume $b_{p}$ replaced by $b_{p}^{\prime}$, where $b_{1}^{\prime}$ and $b_{2}^{\prime}$ are first derivatives of a function $b$. Also we write

$$
b_{p}^{\prime}-\frac{\partial b}{\partial x_{p}}=b_{p}^{\prime \prime}
$$

In equation (2) give $p, q, r$, the values $1,2,3$. It becomes precisely

$$
J\left(\begin{array}{l}
\lambda, b_{3}^{\prime \prime} \\
x_{1}, x_{2}
\end{array}\right)=0
$$

and therefore $b_{3}^{\prime \prime}$ is a function of $Y, x_{3}, x_{4}, \cdots, x_{n}$ only.

We can therefore find a function $F\left(Y, x_{3}, x_{4}, \cdots, x_{n}\right)$ such that $b_{3}^{\prime \prime}=\left(\partial F / \partial x_{3}\right)_{0}$ where the suffix indicates that $Y$ is kept constant. Hence

also

$$
b_{3}^{\prime \prime}=\frac{\partial F}{\partial x_{3}}-\frac{\partial F}{\partial Y} Y_{3}
$$

$$
\frac{\partial F}{\partial x_{1}}=\frac{\partial F}{\partial Y} Y_{1}, \quad \frac{\partial F}{\partial x_{2}}=\frac{\partial F}{\partial Y} Y_{2}
$$


and therefore

$$
\begin{aligned}
& b_{1}=\left(\lambda-\frac{\partial F}{\partial \bar{Y}}\right) Y_{1}+\frac{\partial}{\partial x_{1}}\left(b+F^{\prime}\right), \\
& b_{2}=\left(\lambda-\frac{\partial F}{\partial Y}\right) Y_{2}+\frac{\partial}{\partial x_{2}}\left(b+F^{\prime}\right), \\
& b_{3}=\left(\lambda-\frac{\partial F}{\partial Y}\right) Y_{3}+\frac{\partial}{\partial x_{3}}(b+F),
\end{aligned}
$$

or, changing the notation, we have found functions $b$ and $\lambda$ such that $b_{p}=\lambda Y_{p}+b_{p}^{\prime}$, and $b_{p}^{\prime}=\partial b / \partial x_{p}$ for $p=1,2,3$.

If we now apply (2) for the three sets of values $(1,2,4)$, $(2,3,4),(3,1,4)$, we get

$$
J\left(\begin{array}{c}
Y, b_{4}^{\prime \prime} \\
x_{1}, x_{2}
\end{array}\right)=0, \quad J\left(\begin{array}{c}
Y, b_{4}^{\prime \prime} \\
x_{2}, x_{3}
\end{array}\right)=0, \quad J\left(\begin{array}{c}
Y, b_{4}^{\prime \prime} \\
x_{3}, x_{1}
\end{array}\right)=0,
$$

and hence $b_{4}^{\prime \prime}$ is a function of $Y, x_{4}, x_{5}, \cdots, x_{n}$ only. As before we may modify $\lambda$ and $b$, so as to make $b_{p}^{\prime}=\partial b / \partial x_{4}$ for $p=1,2,3,4$, and the process may be continued so that finally we have

for all values of $p$.

$$
b_{p}=\lambda Y_{p}+\frac{\partial b}{\partial x_{p}}
$$

Again, from (1),

or

$$
\begin{aligned}
\frac{\partial a_{p}}{\partial x_{q}}-\frac{\partial x_{q}}{\partial x_{p}} & =\frac{\partial b}{\partial x_{p}} \frac{\partial Y}{\partial x_{q}}-\frac{\partial b}{\partial x_{q}} \frac{\partial Y}{\partial x_{p}} \\
& =\frac{\partial}{\partial x_{p}}\left(b \frac{\partial Y}{\partial x_{q}}\right)-\frac{\partial}{\partial x_{q}}\left(b \frac{\partial Y}{\partial x_{p}}\right),
\end{aligned}
$$

and therefore

$$
\frac{\partial}{\partial x_{q}}\left(a_{p}+b \frac{\partial Y}{\partial x_{p}}\right)=\frac{\partial}{\partial x_{p}}\left(a_{q}+\frac{\partial Y}{\partial x_{q}}\right)
$$

$$
a_{p}=-b \frac{\partial Y}{\partial x_{p}}+\frac{\partial Z}{\partial x_{p}}
$$

where $Z$ is a new function. The complete solution of (1) is therefore given by

$$
a_{p}=-b \frac{\partial Y}{\partial x_{p}}+\frac{\partial Z}{\partial x_{p}}, \quad b_{p}=\lambda \frac{\partial Y}{\partial x_{p}}+\frac{\partial b}{\partial x_{p}},
$$

where $Z, b, \lambda$, are three arbitrary functions. 
Now consider the equation

$$
\frac{\partial}{\partial x_{r}}\left(H_{p q}\right)+K_{p q} \frac{\partial Y}{\partial x_{r}}=\frac{\partial}{\partial x_{q}}\left(H_{p r}\right)+K_{p r} \frac{\partial Y}{\partial x_{q}} .
$$

Keep $p$ fixed, and let $H_{p q}=a_{q}, K_{p q}=-b_{q^{\circ}}$ We now have equation (1), and hence

$$
\begin{aligned}
& H_{p q}=-B_{p} \frac{\partial Y}{\partial x_{q}}+\frac{\partial Z_{p}}{\partial x_{q}}, \\
& K_{p q}=\lambda_{p} \frac{\partial Y}{\partial x_{q}}+\frac{\partial B_{p}}{\partial x_{q}},
\end{aligned}
$$

where $\lambda_{p}, B_{p}, Z_{p}$, denote $3 n$ as yet arbitrary functions.

Again, $H_{p q}=H_{q p}$, and therefore from (6)

$$
\frac{\partial Z_{p}}{\partial x_{q}}-\frac{\partial Z_{q}}{\partial x_{p}}=B_{p} \frac{\partial Y}{\partial x_{q}}-B_{q} \frac{\partial Y}{\partial x_{p}} .
$$

This equation is of the same type as (1), and hence

$$
Z_{p}=-B \frac{\partial Y}{\partial x_{p}}+\frac{\partial C}{\partial x_{p}}, \quad B_{p}=\nu \frac{\partial Y}{\partial x_{p}}+\frac{\partial B}{\partial x_{p}} .
$$

Similarly from the condition $K_{p q}=K_{q p}$ we have the equations

$$
B_{p}=-\lambda \frac{\partial Y}{\partial x_{p}}+\frac{\partial \eta}{\partial x_{p}}, \quad-\lambda_{p}=-\mu \frac{\partial Y}{\partial x_{p}}+\frac{\partial \lambda}{\partial x_{p}} .
$$

It follows without difficulty that $\nu=-\lambda, \eta=B$, and hence, substituting in (6) and (7) we have the final results

$$
\begin{aligned}
& H_{p q}=\lambda \frac{\partial Y}{\partial x_{p}} \frac{\partial Y}{\partial x_{q}}-\frac{\partial B}{\partial x_{p}} \frac{\partial Y}{\partial x_{q}}-\frac{\partial B}{\partial x_{p}} \frac{\partial Y}{\partial x_{q}}-B \frac{\partial^{2} Y}{\partial x_{p} \partial x_{q}}+\frac{\partial^{2} C^{v}}{\partial x_{p} \partial x_{q} .}, \\
& K_{p q}=\mu \frac{\partial Y}{\partial x_{p}} \frac{\partial Y}{\partial x_{q}}-\frac{\partial \lambda}{\partial x_{p}} \frac{\partial Y}{\partial x_{q}}-\frac{\partial \lambda}{\partial x_{q}} \frac{\partial Y}{\partial x_{p}}-\lambda \frac{\partial^{2} Y}{\partial x_{p} \partial x_{q}}+\frac{\partial^{2} B}{\partial x_{p} \partial x_{q}} .
\end{aligned}
$$

The $n(n+1)$ quantities $H, K$, thus depend on the four arbitrary functions $\lambda, \mu, B, C$.

The above relations may also be written

$$
\begin{aligned}
& H_{p q}=\frac{\partial^{2} A}{\partial x_{p} \partial x_{q}}+Y \frac{\partial^{2} B}{\partial x_{p} \frac{\partial}{\partial x_{q}}+\lambda \frac{\partial Y}{\partial x_{p}} \frac{\partial Y}{\partial x_{q}}} \\
& K_{p q}=\frac{\partial^{2}}{\partial x_{p} \partial x_{q}}(B-\lambda Y)+Y \frac{\partial^{2} \lambda}{\partial x_{p} \partial x_{q}}+\mu \frac{\partial Y}{\partial x_{p}} \frac{\partial Y}{\partial x_{q}} .
\end{aligned}
$$

BRYN MAWR,

$$
\text { May, } 1909 .
$$

